\title{
Cylindrical analogue of NUT space: spacetime of a line gravomagnetic monopole
}

\author{
M. Nouri-Zonoz* \\ Institute of Astronomy, Madingley Road, Cambridge CB3 0HA
}

November 15, 2017

\begin{abstract}
Using the quasi-Maxwell form of the vacuum Einstein equations and demanding the presence of a cylindrically symmetric radial gravomagnetic field, we find the solution to the Einstein equations which represents the gravity field of a line gravomagnetic monopole. We show that this is the generalization of the Levi-Civita's cylindrically symmetric static spacetime, in the same way that the NUT metric is the empty space generalization of the Schwarzschild metric.

Some of the features of this metric as well as its relation to other metrics are discussed.
\end{abstract}

\section{$1 \quad$ Introduction}

Despite their different mathematical structures the analogy between gravitation and electromagnetism has proven to be fruitful. This analogy is particularly useful in the case of gravitation when we try to relate our 3-dimensional physical intuition and experience on the one hand with the 4-dimensional spacetime events on the other hand. It is clear that achiveing this goal requires some sort of splitting of spacetime to space and time components. In this letter we will use this analogy and in particular the quasi-maxwell form of the vacuum Einstein equations to find a solution which corresponds to the presence of a line gravomagnetic monopole. In what follows we will use

${ }^{*}$ Supported by a grant from the ministry of culture and higher education of Iran.

${ }^{1}$ For a discussion on NUT space and gravomagnetic monopoles see [8]. 
the $1+3$ or the threading formulation (splitting) of spacetimes in general relativity introduced by Landau and Lifshitz [6]. In this formulation the fundamental geometrical objects used for splitting spacetime are timelike worldlines (threads) and so the formalism is best suited to treating stationary gravitational fields which are characterized by the existence of a timelike Killing vector.

\section{$1.1 \quad$ The $1+3$ splitting (Threading)}

Suppose that $\mathcal{M}$ is the 4-dimensional manifold of a stationary spacetime with metric $g_{a b}$ and $p \in \mathcal{M}$, then one can show that there is a 3 -dimensional manifold $\Sigma_{3}$ defined invariantly by the smooth map $[1,3]$

$$
\Psi(p): \mathcal{M} \rightarrow \Sigma_{3}
$$

where $\Psi(p)$ denotes the orbit of the timelike Killing vector $\boldsymbol{\xi}_{t}$ passing through $p$. The 3 -space $\Sigma_{3}$ along with the 3 -dimensional metric $\gamma_{\alpha \beta}$ (defined on $\Sigma_{3}$ ) is called the factor space $\frac{(\mathcal{M}, g)}{G_{1}}$, where $G_{1}$ is the 1-dimensional group of transformations generated by $\boldsymbol{\xi}_{t}$. One can use $\gamma_{\alpha \beta}$ to define differential operators on $\Sigma_{3}$ in the same way that $g_{a b}$ defines differential operators on $\mathcal{M}$. For example the covariant derivative of a 3 -vector $\mathbf{A}$ is defined as follows

$$
\begin{aligned}
& A_{; \beta}^{\alpha}=\partial_{\beta} A^{\alpha}+\lambda_{\gamma \beta}^{\alpha} A^{\gamma} \\
& A_{\alpha ; \beta}=\partial_{\beta} A_{\alpha}-\lambda_{\alpha \beta}^{\gamma} A_{\gamma}
\end{aligned}
$$

where $\lambda_{\gamma \beta}^{\alpha}$ is the 3 -dimensional Christoffel symbol cosntructed from the components of $\gamma_{\alpha \beta}$ in the following way

$$
\lambda_{\mu \nu}^{\sigma}=\frac{1}{2} \gamma^{\sigma \eta}\left(\partial_{\nu} \gamma_{\eta \mu}+\partial_{\mu} \gamma_{\eta \nu}-\partial_{\eta} \gamma_{\mu \nu}\right)
$$

It has been shown that the metric of a stationary spacetime can be written in the following form [5]

$$
d s^{2}=e^{-2 \nu}\left(d x^{0}-A_{\alpha} d x^{\alpha}\right)^{2}-d l^{2}
$$

where

$$
A_{\alpha}=\frac{-g_{0 \alpha}}{g_{00}} \quad, \quad e^{-2 \nu} \equiv g_{00}
$$

and

$$
d l^{2}=\gamma_{\alpha \beta} d x^{\alpha} d x^{\beta}=\left(-g_{\alpha \beta}+\frac{g_{0 \alpha} g_{0 \beta}}{g_{00}}\right) d x^{\alpha} d x^{\beta}
$$


is the spatial distance written in terms of the 3 -dimensional metric $\gamma_{\alpha \beta}$ of $\Sigma_{3}$. Using this formulation for a stationary spacetime one can write the vacuum Einstein equations in quasi-Maxwell form as follows [8]

$$
\begin{gathered}
\operatorname{div} \mathbf{B}_{g}=0 \\
\operatorname{Curl} \mathbf{E}_{g}=0 \\
\operatorname{div} \mathbf{E}_{g}=-c^{-2}\left(\frac{1}{2} e^{2 \nu} B_{g}^{2}+E_{g}^{2}\right) \\
\operatorname{Curl}\left(e^{\nu} \mathbf{B}_{g}\right)=-2 c^{-3} \mathbf{E}_{g} \times e^{\nu} \mathbf{B}_{g} \\
P^{\alpha \beta}=E_{g}^{\alpha ; \beta}+e^{2 \nu}\left(B_{g}^{\alpha} B_{g}^{\beta}-B_{g}^{2} \gamma^{\alpha \beta}\right)+E_{g}^{\alpha} E_{g}^{\beta}
\end{gathered}
$$

where the gravitational fields are

$$
\begin{gathered}
\mathbf{E}_{g}=-c^{2} \nabla \nu \\
\mathbf{B}_{g}=c^{2} \text { Curl } \mathbf{A} .
\end{gathered}
$$

and $P^{\alpha \beta}$ is the 3-dimensional Ricci Tensor constructed from the metric $\gamma^{\alpha \beta}$. Note that all operations in these equations are defined in the 3 -dimensional space with metric $\gamma_{\alpha \beta}$.

\section{$1.2 \quad$ Non-relativistic considerations}

Before deriving the spacetime representing a line gravomagnetic monopole, a simple consideration of line magnetic monoploes would be useful. Suppose that a line magnetic monopole is stretched along the $z$-axis in cylindrical coordinates. Using Gauss's law and cylindrical symmetry the $\mathbf{B}$ field produced by this monoploe is given by

$$
\mathbf{B}=\frac{2 Q \hat{\rho}}{\rho}
$$

where $Q=$ const. is the monopole strength per unit length. This field can be produced either by the potential $\mathbf{A}_{1}=\frac{2 Q z}{\rho} \hat{\phi}$ or by $\mathbf{A}_{2}=-2 Q \phi \hat{z}$. The two potentials are related through a gauge transformation

$$
\mathbf{A}_{1}=\mathbf{A}_{2}+\nabla \chi
$$

with $\chi=2 Q z \phi$. Later we will find the gravitational analogue of this gauge transformation. 


\subsection{Generalized Cylindrical solution}

Levi-Civita's static cylindrically symmetric solutions of the vacuum Einstein equations have the following form $[7]$

$$
d s^{2}=\rho^{2 m} d t^{2}-\rho^{-2 m}\left[\rho^{2 m^{2}}\left(d \rho^{2}+d z^{2}\right)+\rho^{2} d \phi^{2}\right]
$$

where $m$ is a constant. 2 To find the stationary cylindrically symmetric solution for empty space (with a radial gravomagnetic field), using equations (1) and (9), we take the following cylindrically symmetric form for the spatial metric

$$
d l^{2}=\gamma_{\alpha \beta} d x^{\alpha} d x^{\beta}=e^{2 \lambda(\rho)}\left(d \rho^{2}+d z^{2}\right)+\rho^{2} e^{2 \nu(\rho)} d \phi^{2}
$$

For cylindrical symmetry with a radial $\mathbf{B}_{g}$, Gauss's law gives

$$
\mathbf{B}_{g}=\frac{L e^{-2 \lambda-\nu}}{\rho} \hat{\rho}
$$

where $L=$ const. is the gravomagnetic monople strength per unit length. Now substituting (7) and (11) into (5a) and (5b) we get the following equation for $\nu(\rho)$ from $(5 \mathrm{a})$

$$
\rho^{2} \nu^{\prime \prime}+\rho \nu^{\prime}-\frac{e^{-4 \nu} L^{2}}{2}=0
$$

while (5b) is satisfied identically. To construct $P^{\alpha \beta}$ from equation (6) we need the 3-dimensional Christoffel symbols of the metric (10) which are

$$
\begin{array}{cc}
\lambda_{\mu \sigma}^{\sigma}=\frac{1}{2 \gamma}\left(\partial_{\mu} \gamma\right) \quad \lambda_{\rho \rho}^{\rho}=\frac{1}{2} \gamma^{\rho \rho}\left(\partial_{\rho} \gamma_{\rho \rho}\right) & \lambda_{z z}^{\rho}=\frac{-1}{2} \gamma^{\rho \rho}\left(\partial_{\rho} \gamma_{z z}\right) \\
\lambda_{z \sigma}^{\tau} \lambda_{z \tau}^{\sigma}=\frac{-1}{2} \gamma^{\rho \rho} \gamma^{z z}\left(\partial_{\rho} \gamma_{z z}\right)^{2} & \lambda_{\phi \sigma}^{\tau} \lambda_{\phi \tau}^{\sigma}=\frac{-1}{2} \gamma^{\rho \rho} \gamma^{\phi \phi}\left(\partial_{\rho} \gamma_{\phi \phi}\right)^{2} \\
\lambda_{\phi \phi}^{\rho}=\frac{-1}{2} \gamma^{\rho \rho}\left(\partial_{\rho} \gamma_{\phi \phi}\right) & \lambda_{\phi \tau}^{\sigma}=\frac{1}{2} \gamma^{\sigma \eta}\left(\partial_{\rho} \gamma_{\eta \tau}\right)
\end{array}
$$

Using the above results and equations (7) and (11), we find that the surviving components of the field equation are

$$
\lambda^{\prime \prime}-\frac{\lambda^{\prime}}{\rho}+2 \nu^{\prime 2}+2 \frac{\nu^{\prime}}{\rho}=0
$$

\footnotetext{
${ }^{2}$ For $m=0,1$ this is a flat metric. Also note that (9) is identical to Kasner solution [4].

${ }^{3}$ The summation convention is being employed here.
} 
and

$$
\rho^{2} \lambda^{\prime \prime}+\rho \lambda^{\prime}-\frac{e^{-4 \nu} L^{2}}{2}=0
$$

where the prime denotes $\frac{\partial}{\partial \rho}$.

Now we proceed to solve equations (12) for $\nu(\rho)$ and $\lambda(\rho)$. Equation (12a) can be written in the following form

$$
\rho \frac{d}{d \rho}\left(\rho \nu^{\prime}\right)=\frac{e^{-4 \nu} L^{2}}{2}
$$

in terms of the variable $u=\ln \rho$ this becomes

$$
\frac{d}{d u}\left(\frac{d \nu}{d u}\right)^{2}=L^{2} e^{-4 \nu} \frac{d \nu}{d u}
$$

which on integration gives the following solution

$$
g_{00} \equiv e^{-2 \nu}=\frac{2 m}{L} \frac{1}{\cosh [2 m \ln (\rho / c)]}=\frac{4 m}{L} \frac{1}{(\rho / c)^{2 m}+(\rho / c)^{-2 m}}
$$

Substituting this into $(12 c)$ one finds

$$
e^{2 \lambda}=a \rho^{2 b} \cosh [2 m \ln (\rho / c)]=a \rho^{2 b} \frac{(\rho / c)^{2 m}+(\rho / c)^{-2 m}}{2}
$$

where $m, a, b$ and $c$ are constants. Substituting (13) and (14) in (12b) one finds that $b=m^{2}$. So writing the metric in form (1), we have

$$
\begin{aligned}
d s^{2}=\frac{2 m}{L} & \frac{1}{\cosh [2 m \ln (\rho / c)]}\left(d t-A_{\alpha} d x^{\alpha}\right)^{2} \\
& -\frac{L}{2 m} \cosh [2 m \ln (\rho / c)]\left[\frac{2 m a}{L} \rho^{2 m^{2}}\left(d \rho^{2}+d z^{2}\right)+\rho^{2} d \phi^{2}\right]
\end{aligned}
$$

To complete the metric we need to choose a potential which produces the field (11) through (8). One can see that the potential expression $\mathbf{A}_{1} \equiv$ $A_{\phi}=L z$ ( $L=$ const.) will do the job 1 . So by substituting this potential expression, the full metric becomes

${ }^{4}$ In a 3 -dimensional space with the metric $\gamma$, the Curl $A$ is defined as follows

$$
(\operatorname{Curl} A)^{\alpha}=\frac{1}{2 \sqrt{\gamma}} e^{\alpha \beta \gamma}\left(\partial_{\beta} A_{\gamma}-\partial_{\gamma} A_{\beta}\right)
$$

where $e_{123}=e^{123}=1$. 


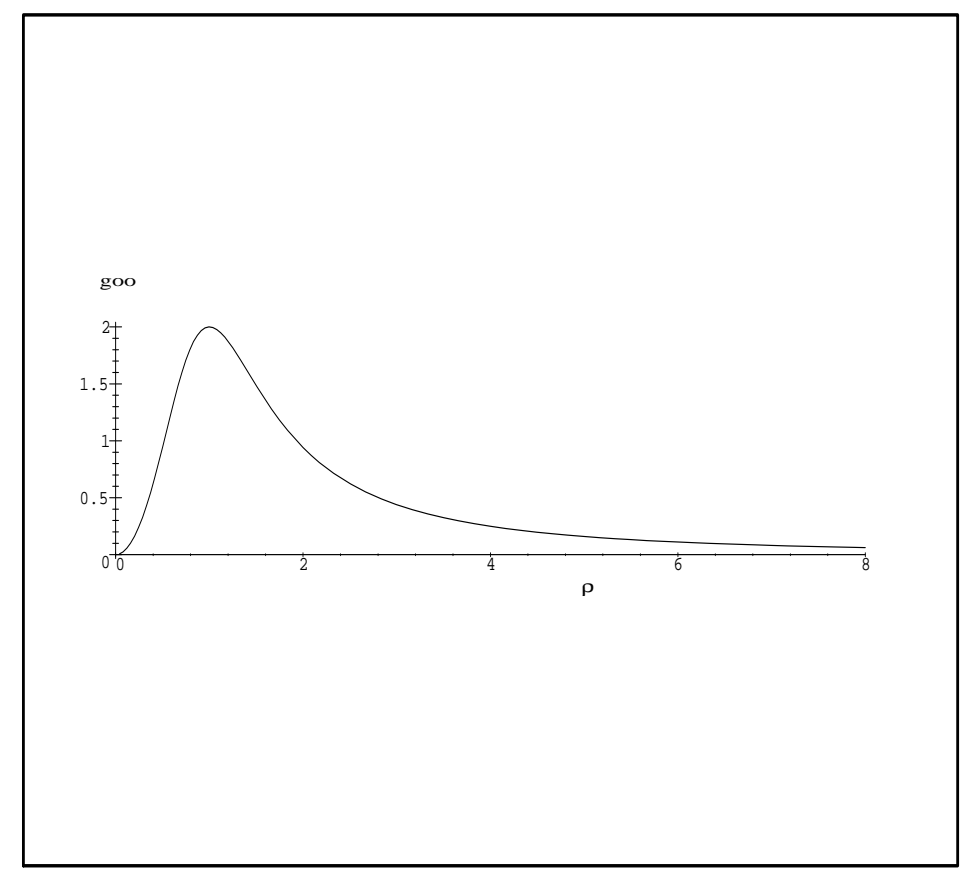

Figure 1: $g_{00}$ as a function of $\rho$ for $L=m=c=1$.

$$
\begin{aligned}
d s^{2}=\frac{2 m}{L} & \frac{1}{\cosh (2 m \ln (\rho / c))}(d t-L z d \phi)^{2} \\
& \quad-\frac{L}{2 m} \cosh (2 m \ln (\rho / c))\left[\frac{2 m a}{L} \rho^{2 m^{2}}\left(d \rho^{2}+d z^{2}\right)+\rho^{2} d \phi^{2}\right]
\end{aligned}
$$

Regaining the static cylindrically symmetric metric as $L \rightarrow 0$ requires that

$$
\begin{gathered}
c=c_{0} L^{\frac{-1}{2 m}} \\
a=\frac{L}{2 m}
\end{gathered}
$$

The behaviour of $g_{00}$ as a function of $\rho$ is shown in figure 1. This spacetime is one of the members of the Papapetrou class [9] and has two event horizons, one at $\rho=0$ and another at $\rho=\infty$. 


\section{2 symmetries}

If we shift the $z$ coordinate in (15a) by a constant $c$ we have

$$
z \rightarrow z^{\prime}=z+c
$$

and

$$
d s^{2} \rightarrow d s^{2}=e^{-2 \nu(\rho)}[d t-(L z+b) d \phi]^{2}-e^{2 \lambda(\rho)}\left(d \rho^{2}+d z^{2}\right)-\rho^{2} e^{2 \nu(\rho)} d \phi^{2}
$$

where $b=L c$. But now one can shift the zero point of the time coordinate in the following way

$$
t \rightarrow t^{\prime}=t-b \phi
$$

so that

$$
d s^{\prime 2}=e^{-2 \nu(\rho)}\left(d t^{\prime}-L z d \phi\right)^{2}-e^{2 \lambda(\rho)}\left(d \rho^{2}+d z^{2}\right)-\rho^{2} e^{2 \nu(\rho)} d \phi^{2}
$$

has the same form as $(15 a)$ in terms of the new coordinate $t^{\prime}$. This shows that $(15 a)$ is translationally invariant along the $z$-direction. In other words, although the metric is not from the mathematical point of view cylindrically symmetric (i.e. not all the components are $z$-independent), it is so physically. This is also clear if one represents the spacetime (15a) by its gravoelectric and gravomagnetic fields which are both explicitly cylindrically symmetric. One can equally well choose as the gravomagnetic potential the expression $\mathbf{A}_{2} \equiv A_{z}=-L \phi$ which leads to the same gravomagnetic field $\mathbf{B}_{g}$. But the metric becomes

$$
\begin{aligned}
d s^{2}=\frac{2 m}{L} & \frac{1}{\cosh (2 m \ln (\rho / c))}(d t+L \phi d z)^{2} \\
& -\frac{L}{2 m} \cosh (2 m \ln (\rho / c))\left[\frac{2 m a}{L} \rho^{2 m^{2}}\left(d \rho^{2}+d z^{2}\right)+\rho^{2} d \phi^{2}\right]
\end{aligned}
$$

This metric is explicitly cylindrically symmetric but it suffers from multivaluedness. If we apply the same reasoning as that we used for the metric (15a) above, we can associate this multivaluedness with different choices of the zero point of the time coordinate through the following transformation

$$
t \rightarrow t^{\prime}=t+b z \quad, \quad b=2 \pi n L \quad n=1,2,3, \ldots
$$

As in the case of the magnetic monopole (section 1.2) the two different choices for the vector potential A correspond to the following gauge transformation between them

$$
\mathbf{A}_{1}=\mathbf{A}_{2}+\nabla \chi
$$

where $\chi=L z \phi$. 


\section{$2.1 \quad$ Killing vectors}

It is already clear that $\boldsymbol{\xi}_{t}=(1,0,0,0)$ and $\boldsymbol{\xi}_{\phi}=(0,0,0,1)$ are killing vectors of $(15 a)$ however one can see that the form $(15 b)$ can be reached alternatively by the transformation $t \rightarrow t^{\prime}=t-L z \phi$. Now, as is obvious from (15b), $\xi_{z}^{\prime \beta}=(0,0,1,0)$ is a Killing vector in the transformed coordinates and upon converting to the previous coordinates we find that

$$
\xi^{\alpha}=\frac{\partial x^{\alpha}}{\partial x^{\prime \beta}} \xi^{\prime \beta}=\left(\frac{\partial t}{\partial z^{\prime}}, 0, \frac{\partial z}{\partial z^{\prime}}, 0\right)=(L \phi, 0,1,0)
$$

is also a Killing vector of (15). 9 This is a multivalued Killing vector whose interpretation might require the consideration of not just the space itself but also the covering space with the many values of $\phi$ unroled.

\section{$3 \quad$ Relation to other metrics}

To find the connection between this metric and some other solutions, we use the Ernst formulation of axially symmetric spacetimes. In this formulation the vacuum field equations, in the Weyl's coordinates $(\rho, z)$, f can be written in the following form $[2,5]$

$$
\left(\varepsilon+\varepsilon^{*}\right)\left(\varepsilon_{, \rho \rho}+\rho^{-1} \varepsilon_{, \rho}+\varepsilon_{, z z}\right)=2\left(\varepsilon_{, \rho}^{2}+\varepsilon_{, z}^{2}\right)
$$

where $\varepsilon$ is a complex potential defined as follows

$$
\varepsilon=e^{2 U}+i \omega \quad, \quad e^{2 U}=g_{00}
$$

Every solution of (16) gives a stationary axially symmetric metric. one can easily see that the metric $(15 a)$ is a solution of (16) with

$$
e^{2 U}=g_{00}=\frac{2 m}{L} \frac{1}{\cosh (2 m \ln (\rho / c))}
$$

and

$$
\omega=\frac{2 m}{L} \tanh (2 m \ln (\rho / c))
$$

\footnotetext{
${ }^{5}$ I am grateful to D. Lynden-Bell and J. Katz for pointing out this to me.

${ }^{6}$ In these coordinates the metric of an axially symmetric stationary spacetime can be written in the following form

$$
d s^{2}=e^{2 U}(d t+A d \phi)^{2}-e^{-2 U}\left[e^{2 K}\left(d \rho^{2}+d z^{2}\right)+\rho^{2} d \phi^{2}\right] .
$$
}


Now the following theorems lead us to some other solutions [5]

$(I)$. Given a stationary axisymmetric vacuum solution $\left(\varepsilon=e^{2 U}+i \omega\right)$, the substitution

$$
\begin{gathered}
U^{\prime}=-U+\frac{1}{2} \ln \rho \\
\omega^{\prime}=i \omega
\end{gathered}
$$

yields another vacuum solution $\left(U^{\prime}, \omega^{\prime}\right)$.

$(I I)$. and the substitution

$$
\begin{aligned}
& U^{\prime}=2 U \\
& \omega^{\prime}=i \omega
\end{aligned}
$$

yields a solution $\left(U^{\prime}, \omega^{\prime}\right)$ of the Einstein-Maxwell equations.

$(I I I)$. For a stationary axisymmetric vacuum solution $(U, \omega)$, (in Weyl's coordinates) one obtains a corresponding cylindrically symmetric EinsteinMaxwell field $\left(U^{\prime}, \omega^{\prime}\right)$ by the substitution

$$
t \rightarrow i z \quad z \rightarrow i t \quad 2 U \rightarrow U^{\prime} \quad \omega \rightarrow \omega^{\prime}
$$

For example applying (I) followed by (III) to (15a), one obtains the Rahdakrishna metric [10], which is a time dependent cylindrically symmetric solution of Einstein-Maxwell equations.

\subsection{Gravitational duality rotation}

One can also obtain metric (15) by applying the gravitational duality rotation (sometimes called Ehlers' transformation)]to metric (9). This transformation, in its original formulation, states that if

$$
g_{\mu \nu}=e^{2 U}\left(d x^{0}\right)^{2}-e^{-2 U} d l^{2}
$$

is the metric of a static exterior spacetime, then

$$
\bar{g}_{\mu \nu}=(a \cosh (2 U))^{-1}\left(d x^{0}-A_{\alpha} d x^{\alpha}\right)^{2}-a \cosh (2 U) d l^{2}
$$

[with $a=$ const. $>0, U=U\left(x^{\alpha}\right)$ and $A_{\beta}=A_{\beta}\left(x^{\alpha}\right)$ ] would be the metric of a stationary exterior spacetime provided that $A_{\alpha}$ satisfies

$$
-a \sqrt{\gamma} \epsilon_{\alpha \beta \eta} U^{, \eta}=A_{[\alpha, \beta]}
$$

\footnotetext{
${ }^{7}$ This transformation was first introduced by Ehlers [1] and later developed by Geroch $[3]$.
} 
where $\epsilon_{\alpha \beta \gamma}$ is the flat space alternating symbol. From equations (9) and (15a) we see that in this case

$$
\begin{gathered}
a=\frac{L}{2 m} \\
A_{\alpha} \equiv A_{\phi}=L_{z} \\
U=m \ln \rho
\end{gathered}
$$

and

$$
d l^{2}=\rho^{2 m^{2}}\left(d \rho^{2}+d z^{2}\right)+\rho^{2} d \phi^{2}
$$

It can easily be seen that equations (19) satisfy (18). In the same way the NUT metric has been shown to be the gravitational dual of Schwarzschild metric [3].

\section{ACKNOWLEDGEMENTS}

I would like to thank my supervisor, Professor D. Lynden-Bell, without whose help this work would not have been done. I am also grateful to Dr. H. Ardavan for useful discussions and comments.

\section{References}

[1] Ehlers, J., Colloques internationaux C.N.R.S. No. 91 ( Les theories relativistes de la gravitation), 275, 1962.

[2] Ernst, F. J., Phys.Rev.,167, 1175, 1968; 168, 1415, 1968.

[3] Geroch, R., J.Math.Phys., 12, 918, 1972.

[4] Kasner, E., Amer. J. Math. 43, 217, 1921.

[5] D. Kramer, H. Stephani, E. Herlt and M. MacCallum, Exact Solutions of Einstein's Field Equations, Ed. E. Schmutzer, 1980, CUP.

[6] L. D. Landau and E. M. Lifishitz, classical theory of fields., 4th edn. Pergamon press, Oxford, 1975.

[7] Levi-Civita, T., Rend.acc. Lince; 26, 307, 1917.

[8] D. Lynden-Bell and M. Nouri-Zonoz, gr-qc/9612049. 
[9] A. Papapetrou., Annalen Physik; 12, 309, 1953.

[10] Radhakrishna, L., Proc.Nat.Inst.India, A29, 588, 1968. 